\title{
CARDINAL INTERPOLATION BY SYMMETRIC EXPONENTIAL BOX SPLINES ON A THREE-DIRECTION MESH
}

\author{
by T. N. T. GOODMAN and A. A. TAANI
}

(Received 9th January 1989)

\begin{abstract}
We consider certain exponential box splines $E$ on a three-direction mesh whose exponents satisfy a symmetry condition. It is shown, in particular, that given bounded data on the integer lattice in $\mathbf{R}^{2}$, there is a unique bounded combination of integer translates of $E$ that interpolates the data. When all exponents are zero, this reduces to a result of de Boor, Höllig and Riemenschneider in [2]. Unlike the proof in [2] we use only elementary analysis and do not employ any computer calculations.
\end{abstract}

1980 Mathematics subject classification (1985 Revision): 41A05, 41 A63.

\section{Introduction}

Let $v$ be a positive integer and consider the elements of Euclidean space $\mathbf{R}^{v}$ as column vectors. In [5] Ron defines exponential box splines ( $E$-splines) as follows. Let $\Omega$ be a finite sequence, called a defining sequence, whose elements are of the form $\omega=\left(x_{\omega}, \lambda_{\omega}\right)$, where $x_{\omega} \in \mathbf{R}^{\nu}-\{0\}, \lambda_{\omega} \in \mathbf{R}$. We define $X_{\Omega^{*}}=\left\{x_{\omega}: \omega \in \Omega\right\}$ which can be regarded as a $v \times|\Omega|$ matrix, and $\Lambda_{\Omega}:=\left\{\lambda_{\omega}: \omega \in \Omega\right\} \in \mathbf{R}^{|\Omega|}$. Then the $E$-spline $E=E(\Omega \mid \cdot)$ related to $\Omega$ is a distribution on $\mathbf{R}^{\mathbf{v}}$ defined by the requirement:

$$
\int_{\mathbf{R}^{\nu}} E(\Omega \mid x) f(x) d x=\int_{[0,1]} \exp \left(\Lambda_{\Omega} t\right) f\left(X_{\Omega} t\right) d t, \quad f \in C_{0}^{\infty}\left(\mathbf{R}^{v}\right)
$$

It is clear from (1.1) that if $\Lambda_{\Omega}$ is a zero sequence, then $E(\Omega \mid \cdot)$ reduces to the box spline $B\left(X_{\Omega} \mid \cdot\right)$ based on the sequence $X_{\Omega}$ studied in $[1,4]$ and elsewhere.

We shall find it convenient to change definition (1.1) by making a translation of coordinate system so that the origin is the centre of the support of $E(\Omega \mid \cdot)$. Thus we use the definition:

$$
\int_{\mathbf{R}^{\nu}} E(\Omega \mid x) f(x) d x=\int_{[-1 / 2,1 / 2]^{|\Omega|}} \exp \left(\Lambda_{\mathbf{\Omega}} t\right) f\left(X_{\mathbf{\Omega}} t\right) d t, \quad f \in C_{0}^{\infty}\left(\mathbf{R}^{v}\right)
$$

The Fourier transform of $\hat{E}(\Omega \mid \cdot)$ is given by:

$$
\hat{E}(\Omega \mid x)=\prod_{\omega \in \Omega} \frac{\exp \frac{1}{2}\left(\lambda_{\omega}-i x^{*} x_{\omega}\right)-\exp \frac{1}{2}\left(i x^{*} x_{\omega}-\lambda_{\omega}\right)}{\lambda_{\omega}-i x^{*} x_{\omega}},
$$

where $x^{*}$ denotes the transpose of $x$. 
A defining sequence $\Omega$ is called symmetric if $\left\{\left(x_{\omega},-\lambda_{\omega}\right): \omega \in \Omega\right\}$ is a rearrangement of $\Omega$. The $E$-spline $E(\Omega \mid \cdot)$ is called symmetric if $\Omega$ is symmetric. In this case (1.3) clearly shows that $\hat{E}(\Omega \mid x)=\hat{E}(\Omega \mid-x)$ and hence

$$
E(\Omega \mid x)=E(\Omega \mid-x)
$$

For any $v \times v$ non-singular matrix $A$ we define

$$
A \Omega:=\left\{\left(A x_{\omega}, \lambda_{\omega}\right): \omega \in \Omega\right\}
$$

Then it follows from (1.3) that for $x$ in $\mathbf{R}^{v}$,

$$
\hat{E}(A \Omega \mid x)=\hat{E}\left(\Omega \mid A^{*} x\right)
$$

and so

$$
E(A \Omega \mid A x)|\operatorname{det} A|=E(\Omega \mid x)
$$

For a function $\phi$ with compact support on $\mathbf{R}^{v}$, denote by

$$
S(\phi):=\operatorname{span}\left\{\phi(\cdot-j): j \in \mathbf{Z}^{v}\right\}
$$

the space generated by integer translates of $\phi$. So the elements of $S(\phi)$ have the form

$$
s(\cdot)=\sum_{j \in \mathbf{Z}^{v}} a_{j} \phi(: \div j)
$$

The cardinal interpolation problem for $\phi$ can be stated as follows. For any $1 \leqq p \leqq \infty$ and data sequence $F=\left\{f(k): k \in \mathbf{Z}^{v}\right\}$ in $l_{p}\left(\mathbf{Z}^{v}\right)$, determine a coefficient sequence $a=$ $\left\{a_{j}: j \in \mathbf{Z}^{v}\right\}$ in $l_{p}\left(\mathbf{Z}^{v}\right)$ such that

$$
s_{f}(x):=\sum_{j \in \mathbf{Z}^{v}} a_{j} \phi(x-j) \in S(\phi)
$$

interpolates the data sequence $F$, i.e.,

$$
s_{f}(k)=f(k), \quad k \in \mathbf{Z}^{v}
$$

If such a coefficient sequence always exists and is unique we say the cardinal interpolation problem is correct. It is shown in [2] that the cardinal interpolation problem for $\phi$ is correct if and only if the trigonometric polynomial

$$
\sum_{j \in \mathbf{Z}^{v}} \phi(j) e^{i j x}, \quad x \in \mathbf{R}^{v}
$$


does not vanish. In particular the cardinal interpolation problem for $E(\Omega \mid \cdot)$ is correct iff

$$
\tilde{E}(x)=\tilde{E}(\Omega \mid x):=\sum_{j \in \mathbf{Z}^{v}} E(\Omega \mid j) e^{i j x}, \quad x \in \mathbf{R}^{v},
$$

does not vanish. This polynomial is called the discrete Fourier transform of $E(\cdot)$ (see [3]). By applying the Poisson summation formula to (1.7) we see that:

$$
\tilde{E}(x)=\sum_{j \in \mathbf{Z}^{v}} \hat{E}(2 \pi j-x)
$$

In Section 3 we consider the situation when $\nu=2, \Omega$ is symmetric and $X_{\Omega}$ comprises at most three distinct vectors. After studying some symmetry properties of $E$ we proceed in Section 4 to show from (1.8) that $\tilde{E}$ is strictly positive and hence the cardinal interpolation problem for $E$ is correct. When $\Lambda_{\Omega}$ is a zero sequence, this result reduces to a result of de Boor, Hollig and Riemenschneider in [2]. Unlike the proof in [2] we do not employ any computer calculations and the tensor product case (at most two distinct vectors in $X_{\Omega}$ ) does not require a separate proof. For clarity we prove the univariate case (all vectors in $X_{\Omega}$ equal) separately in Section 2 but this result is not used in later sections.

\section{Univariate cardinal interpolation}

Univariate cardinal interpolation with a symmetric $E$-spline is essentially a special case of the result for a three-direction mesh which we shall discuss in the rest of the paper. However we consider it separately in this section to illustrate the method in this simple case when it is not obscured by the more complicated details of the bivariate case.

Theorem 1. If $\Omega=\left\{\left(1, \lambda_{1}\right), \ldots,\left(1, \lambda_{r}\right)\right\}$ is a symmetric defining sequence, then the cardinal interpolation problem for $E(\Omega \mid \cdot)$ is correct.

Proof. We shall show that the discrete Fourier transform

$$
\tilde{E}(x):=\sum_{j=-\infty}^{\infty} E(j) e^{i j x}, \quad x \in \mathbf{R}
$$

is strictly positive. Since $\tilde{E}$ is periodic of $2 \pi$, it is sufficient to show that $\tilde{E}>0$ on $[-\pi, \pi]$. Furthermore $E$ is an even function, by (1.4), and so it is enough to show that $\widetilde{E}(x)>0$ for $0 \leqq x \leqq \pi$. Suppose that $\Lambda_{\Omega}$ has $\rho$ zero elements and $P:=\{\lambda \in \Lambda: \lambda>0\}$. Then from (1.3) and (1.8),

$$
\tilde{E}(x)=\sum_{-\infty}^{\infty} g_{j}(x), \quad 0 \leqq x \leqq \pi
$$


where

$$
g_{j}(x)=\left[\frac{(-1)^{j} \sin (x / 2)}{j \pi+(x / 2)}\right]^{\rho} \prod_{\lambda \in P} \frac{e^{\lambda}+e^{-\lambda}-2 \cos x}{\lambda^{2}+[2 \pi j+x]^{2}}, \quad 0 \leqq x \leqq \pi .
$$

We consider two cases

Case 1. $\rho$ is even. Here $g_{j} \geqq 0$ for $j \in \mathbf{Z}$ and $g_{0}>0$.

Case 2. $\rho$ is odd. Now $\left|g_{0}\right|>\left|g_{1}\right|$ and $\left|g_{j}\right| \geqq\left|g_{j+1}\right|, j=1,2,3, \ldots$ Since $(-1)^{j} g_{j} \geqq 0$ for $j \in \mathbf{Z}$ we have

$$
g_{0}+g_{1}>0, \quad g_{2 j}+g_{2 j+1} \geqq 0, \quad j=1,2,3, \ldots
$$

Similarly

$$
g_{-2 j-1}+g_{-2 j-2} \geqq 0, \quad j=1,2,3, \ldots
$$

Thus from $(2.1), \tilde{E}(x)>0,0 \leqq x \leqq \pi$.

\section{Symmetric $E$-splines on a three-direction mesh}

For the rest of this paper we are concerned with the case $v=2$ and the situation where $X_{\Omega}$ comprises only three distinct vectors: $e_{1}=(1,0), e_{2}=(0,1), e_{3}=e_{1}+e_{2}$.

Thus the defining sequence $\Omega$ has the form

$$
\Omega=\left\{\left(e_{1}, \lambda_{1}^{1}\right), \ldots,\left(e_{1}, \lambda_{r}^{1}\right),\left(e_{2}, \lambda_{1}^{2}\right), \ldots,\left(e_{2}, \lambda_{s}^{2}\right),\left(e_{3}, \lambda_{1}^{3}\right), \ldots,\left(e_{3}, \lambda_{t}^{3}\right)\right\}
$$

We shall assume $\Omega$ is symmetric. For $i=1,2,3$, we define $P_{i}:=\left\{\lambda_{j}^{i}: \lambda_{j}^{i}>0\right\}$ and let $\rho_{i}$ denote the number of elements in $\left\{\lambda_{j}^{i} \cdot \lambda_{j}^{i}=0\right\}$. Then from (1.3) we have

$$
\hat{E}(\Omega \mid(u, v))=g^{1}(u) g^{2}(v) g^{3}(u+v),
$$

where for $i=1,2,3$

$$
g^{i}(t)=\left[\frac{\sin (t / 2)}{t / 2}\right]^{\rho_{i}} \prod_{\lambda \in P_{i}} \frac{e^{\lambda}+e^{-\lambda}-2 \cos t}{\lambda^{2}+t^{2}}
$$

We now deduce some symmetry properties, following the method of [2]. We first note that if $\Omega$ is obtained from $\Omega$ (of (3.1)) by replacing $e_{1}$ and/or $e_{2}$ and/or $e_{3}$ by their negative, then

$$
E(\widetilde{\Omega} \mid \cdot)=E(\Omega \mid \cdot)
$$

For any permutation $\sigma$ in $S_{3}$ (the symmetric group on three elements) we define 


$$
\sigma(\Omega)=\left\{\left(e_{\sigma(1)}, \lambda_{1}^{1}\right), \ldots,\left(e_{\sigma(1)}, \lambda_{r}^{1}\right),\left(e_{\sigma(2)}, \lambda_{1}^{2}\right), \ldots,\left(e_{\sigma(2)}, \lambda_{s}^{2}\right),\left(e_{\sigma(3)}, \lambda_{1}^{3}\right), \ldots,\left(e_{\sigma(3)}, \lambda_{t}^{3}\right)\right\}
$$

We now construct a homomorphism $\sigma \mapsto A_{\sigma}$ from $S_{3}$ into the group of non-singular $2 \times 2$ matrices by defining for the transpositions (12), (13), (23):

$$
A_{(12)}:=\left[\begin{array}{cc}
0 & -1 \\
-1 & 0
\end{array}\right], \quad A_{(13)}:=\left[\begin{array}{cc}
1 & 0 \\
1 & -1
\end{array}\right], \quad A_{(23)}:=\left[\begin{array}{cc}
-1 & 1 \\
0 & 1
\end{array}\right] .
$$

We note that for any $\sigma$ in $S_{3}$ and $1 \leqq i \leqq 3$,

$$
A_{\sigma} e_{i} \in\left\{e_{\sigma(i)},-e_{\sigma(i)}\right\} \text {. }
$$

It follows from (3.7), (3.5) and (3.4) that for any $\sigma$ in $S_{3}$,

$$
E(\sigma(\Omega) \mid \cdot)=E\left(A_{\sigma} \Omega \mid \cdot\right),
$$

and so from (1.4) and (1.5),

$$
E(\Omega \mid x)=E\left(\sigma(\Omega) \mid \pm A_{\sigma} x\right) .
$$

From (3.9) and (1.7) we see that for $\sigma$ in $S_{3}$,

$$
\tilde{E}(\sigma(\Omega) \mid x)=\tilde{E}\left(\Omega \mid \pm A_{\sigma}^{*} x\right), \quad x \in \mathbf{R}^{2} .
$$

\section{Cardinal interpolation on a three-direction mesh}

We continue with our notation of Section 3 and we now prove our main result:

Theorem 2. The cardinal interpolation problem for a symmetric E-spline on a threedirection mesh is correct.

Proof. We shall show that for $x$ in $\mathbf{R}^{2}$,

$$
\tilde{E}(\Omega \mid x)>0 .
$$

Since $\tilde{E}(\Omega \mid \cdot)$ is periodic of period $2 \pi$, it is sufficient to show (4.1) for $x$ in $[-\pi, \pi]^{2}$. Now it is easily seen that

$$
[-\pi, \pi]^{2} \subseteq \bigcup_{\sigma \in S_{3}} A_{\sigma}^{*}[0, \pi]^{2}
$$

So from (3.10) it is sufficient to prove (4.1) for $x$ in $[0, \pi]^{2}$. Now by (1.8), (3.2), and (3.3), inequality (4.1) for $x$ in $[0, \pi]^{2}$ is equivalent to 


$$
T(u, v):=\sum_{k, l \in \mathbf{Z}} G_{k}^{1}(u) G_{l}^{2}(v) G_{k+l}^{3}(u+v)>0, \quad u, v \in\left[0, \frac{1}{2}\right]
$$

where for $i=1,2,3, j \in \mathbf{Z}$,

$$
G_{j}^{i}(t)=\left[\frac{(-1)^{j} \sin \pi t}{j+t}\right]^{\rho_{i}} \prod_{2 \pi \lambda \in P_{i}} \frac{1}{\lambda^{2}+(j+t)^{2}}, \quad 0 \leqq t \leqq 1 .
$$

It will be convenient to write, for $i, j$ in $\mathbf{Z}$,

$$
G_{i j}(u, v):=G_{i}^{1}(u) G_{j}^{2}(v) G_{i+j}^{3}(u+v), \quad u, v \in\left[0, \frac{1}{2}\right]
$$

We divide the proof into eight cases depending on the parity of $r, s$ and $t$, or equivalently of $\rho_{1}, \rho_{2}$ and $\rho_{3}$

Case 1. $r, s$ and $t$ even. Here $G_{k l} \geqq 0$ for $k, l$ in $\mathbf{Z}$. Moreover $G_{00}(u, v)>0$ unless $u=v=\frac{1}{2}$, and $G_{-1,0}\left(\frac{1}{2}, \frac{1}{2}\right)>0$. Thus

$$
T=\sum_{k, l \in \mathbf{Z}} G_{k l}>0
$$

For the remaining cases we shall repeatedly use the facts that for $i=1,2,3$,

$$
\begin{array}{ll}
\left|G_{j}^{i}(t)\right| \geqq\left|G_{j+1}^{i}(t)\right|, & 0 \leqq t \leqq 1, \quad j \geqq 0, \\
\left|G_{j}^{i}(t)\right| \geqq\left|G_{j-1}^{i}(t)\right|, \quad 0 \leqq t \leqq 1, \quad j<0,
\end{array}
$$

with strict inequality unless the terms vanish.

Case 2. $r$ odd, $s$ and $t$ even. We shall deduce some inequalities for $G_{i j}$ from (4.5) and (4.4). The appropriate regions of $(i, j)$ are illustrated in Figure 1.

We see that

$$
\begin{aligned}
G_{2 k, l}+G_{2 k+1, l} \geqq 0, & k \geqq 0, \quad l>0 \quad(\text { region A), } \\
G_{2 k+1, l}+G_{2 k, l} \geqq 0, & k<0, \quad l \leqq 0 \quad \text { (region } \mathrm{B}), \\
G_{2 k, l}+G_{2 k+1, l-1} \geqq 0, & k \geqq 0, \quad 2 k+l<0 \quad \text { (region C), } \\
G_{2 k+1, l-1}+G_{2 k, l} \geqq 0, & k<0, \quad 2 k+l>0 \quad \text { (region D). }
\end{aligned}
$$

Next we show that (see region E)

$$
G_{2 k, 2 l}+G_{2 k+1,2 l-1}+G_{2 k+1,2 l}+G_{2 k+2,2 l-1} \geqq 0, \quad l \leqq 0, \quad k+l \geqq 0 .
$$

Now (4.10) is equivalent to 


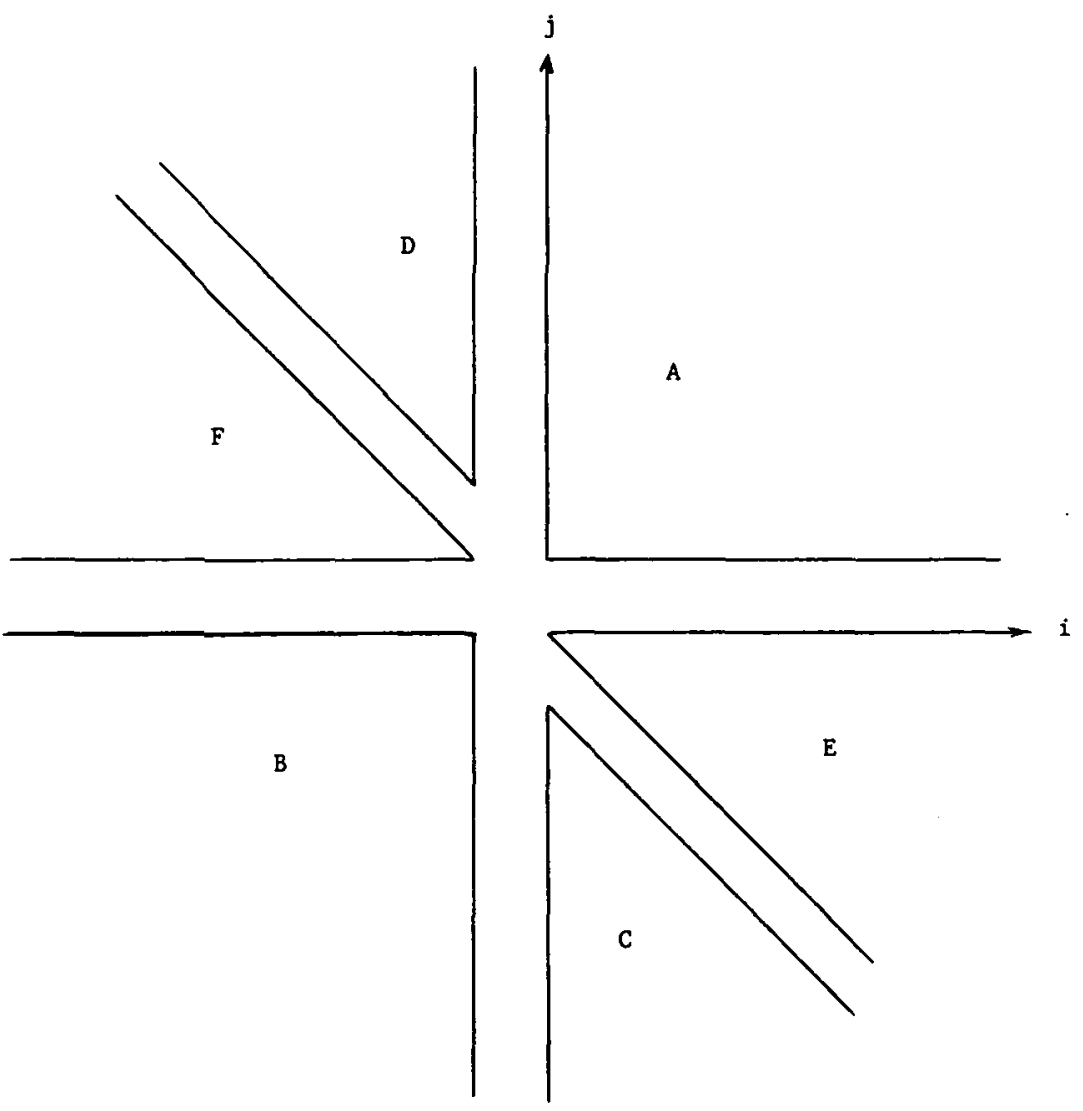

FIGURE 1 Regions for $G_{i j}$.

$$
\left|G_{2 k, 2 l}\right|-\left|G_{2 k+1,2 l-1}\right| \geqq\left|G_{2 k+1,2 l}\right|-\left|G_{2 k+2,2 l-1}\right|
$$

From (4.4),

$$
\begin{aligned}
& \left|G_{2 k, 2 l}(u, v)\right|-\left|G_{2 k+1,2 l-1}(u, v)\right| \\
& \left.\quad=G_{2 k+2 l}^{3}(u+v)\left\{G_{2 k}^{1}(u)\left(G_{2 l}^{2}(v)-G_{2 l-1}^{2}(v)\right)+\left(\left|G_{2 k}^{1}(u)\right|-\left|G_{2 k+1}^{1}(u)\right|\right) G_{2 l-1}^{2}(v)\right)\right\}
\end{aligned}
$$

Since for $l \leqq 0, k+l \geqq 0, G_{2 k+2 l}^{2}(u+v)$ and $G_{2 k}^{1}(u)$ are decreasing functions of $k$ and $G_{2 l}^{2}(v)-G_{2 l-1}^{2}(v) \geqq 0$, to prove (4.1) it suffices to show that

$$
\left|G_{2 k}^{1}(u)\right|-\left|G_{2 k+1}^{1}(u)\right|
$$

is a decreasing function of $k$ for $k \geqq 0$. Now writing $P_{1}=\left\{2 \pi \lambda_{1}, \ldots, 2 \pi \lambda_{n}\right\}$ and $\left|G_{k}^{1}(u)\right|-$ $\left|G_{k+1}^{1}(u)\right|=(\sin \pi u)^{\rho_{1}} f(k)$ we have 


$$
\begin{aligned}
f(k)= & \frac{1}{(k+u)^{\rho_{1}}} \prod_{i=1}^{n} \frac{1}{\lambda_{i}^{2}+(k+u)^{2}}-\frac{1}{(k+1+u)^{\rho_{1}}} \prod_{i=1}^{n} \frac{1}{\lambda_{i}^{2}+(k+1+u)^{2}} \\
= & \frac{1}{(k+u)^{\rho_{1}}} \prod_{i=1}^{n-1} \frac{1}{\lambda_{i}^{2}+(k+u)^{2}}\left\{\frac{1}{\lambda_{n}^{2}+(k+u)^{2}}-\frac{1}{\lambda_{n}^{2}+(k+1+u)^{2}}\right\}+\cdots \\
& +\left(\frac{1}{k+u}-\frac{1}{k+1+u}\right) \frac{1}{(k+1+u)^{\rho_{1}-1}} \prod_{i=1}^{n} \frac{1}{\lambda_{i}^{2}+(k+1+u)^{2}} \\
= & \frac{2 k+2 u+1}{(k+u)^{\rho_{1}}} \prod_{i=1}^{n} \frac{1}{\lambda_{i}^{2}+(k+u)^{2}} \frac{1}{\lambda_{i}^{2}+(k+1+u)^{2}}+\cdots \\
& +\frac{1}{k+u} \frac{1}{(k+1+u)^{\rho_{1}}} \prod_{i=1}^{n} \frac{1}{\lambda_{i}^{2}+(k+1+u)^{2}},
\end{aligned}
$$

which is a decreasing function of $k$ for $k \geqq 0, u \geqq 0$. Thus (4.12) is decreasing in $k$ which gives (4.11) and hence (4.10).

Similarly, we can show that

$$
G_{2 k+1,2 l-1}+G_{2 k, 2 l}+G_{2 k, 2 l-1}+G_{2 k-1,2 l} \geqq 0, \quad l \geqq 1, \quad k+l \leqq 0 \quad \text { (region F). (4.13) }
$$

From (4.6)-(4.10) and (4.13) we can see that $T \geqq 0$ in (4.2). Also we have strict inequality in (4.10) for $k=l=0$ unless $u=v=\frac{1}{2}$, and strict inequality in (4.7) for $k=-1$, $l=0, u=v=\frac{1}{2}$. Thus $T>0$ in (4.2).

Case 3. $s$ odd, $r$ and $t$ even. This follows from Case 2 by symmetry.

Case 4. $t$ odd, $r$ and $s$ even. We shall deduce some inequalities for $G_{i j}$ from (4.5) and (4.4). The appropriate regions of $(i, j)$ are illustrated in Figure 2.

We see that

$$
\begin{aligned}
& G_{2 k-l, l}+G_{2 k+1-l, l} \geqq 0, \quad k>0, \quad l<0 \quad \text { (region A), } \\
& G_{2 k+1-l, l}+G_{2 k-l, l} \geqq 0, \quad k<0, \quad l>0 \quad \text { (region } \mathrm{B} \text { ), } \\
& G_{k, 2 l+1-k}+G_{k, 2 l-k} \geqq 0, \quad k \geqq 0, \quad l<0 \quad \text { (region } \mathrm{C} \text { ), } \\
& G_{k, 2 l-k}+G_{k, 2 l+1-k} \geqq 0, \quad k<0, \quad l \geqq 0 \quad \text { (region D). }
\end{aligned}
$$

Following the derivation of $(4.10)$ we can see that

$$
G_{2 k, 2 l}+G_{2 k, 2 l+1}+G_{2 k+1,2 l}+G_{2 k+1,2 l+1} \geqq 0, \quad k, l \geqq 0 \quad \text { (region E), }
$$




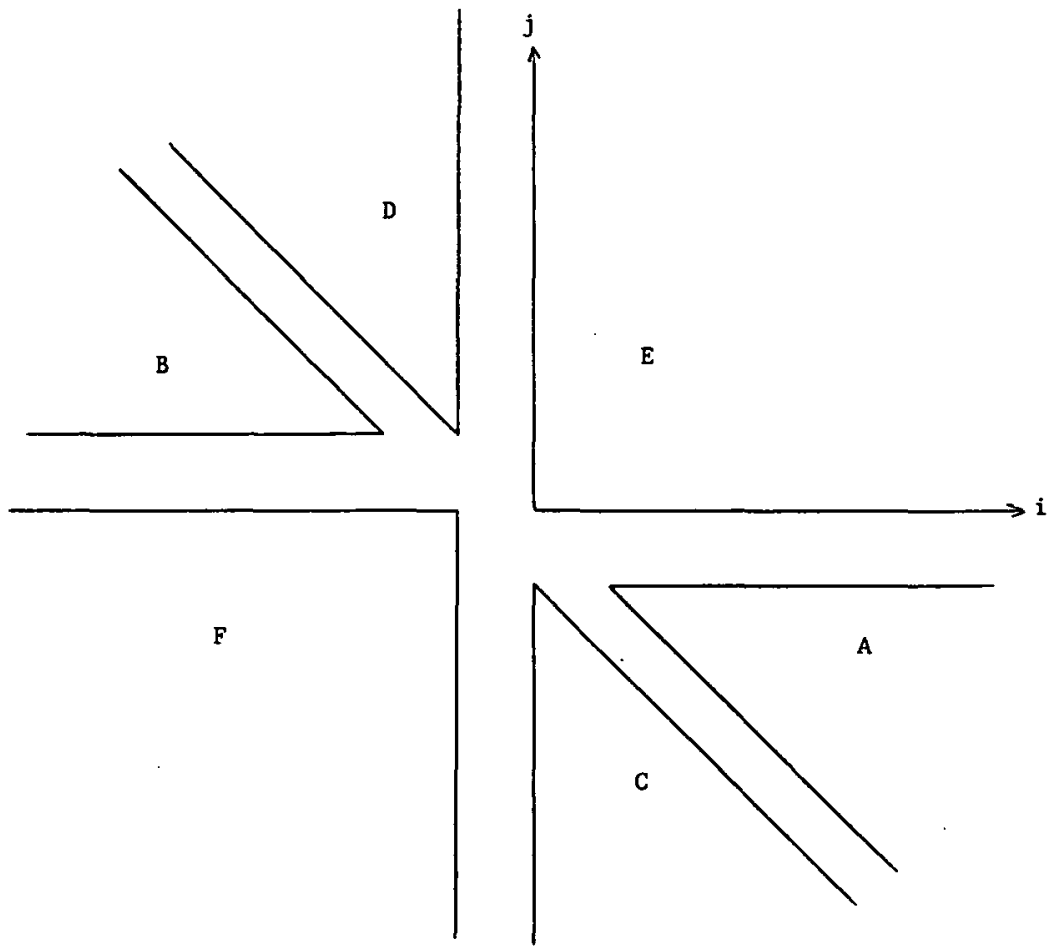

FIGURE 2 Regions for $G_{i j}$.

$G_{2 k-1,2 l}+G_{2 k-1,2 l-1}+\dot{G}_{2 k-2,2 l}+G_{2 k-2,2 l-1} \geqq 0, \quad k, l \leqq 0 \quad$ (region $\mathrm{F}$ ).

From (4.14)-(4.19) we have $T \geqq 0$. Here and in the remaining cases the strict inequality $T>0$ follows from considering appropriate choices of the above inequalities for different values of $u$ and $v$ as in Case 2 .

Case 5. $r$ and $s$ odd, $t$ even. We shall deduce some inequalities for $G_{i j}$. The appropriate regions of $(i, j)$ are illustrated in Figure 3.

We first claim that

$$
\sum_{-\infty}^{\infty} G_{j,-j} \geqq 0
$$

For from (4.3) we have for $i=1,2,3$ 


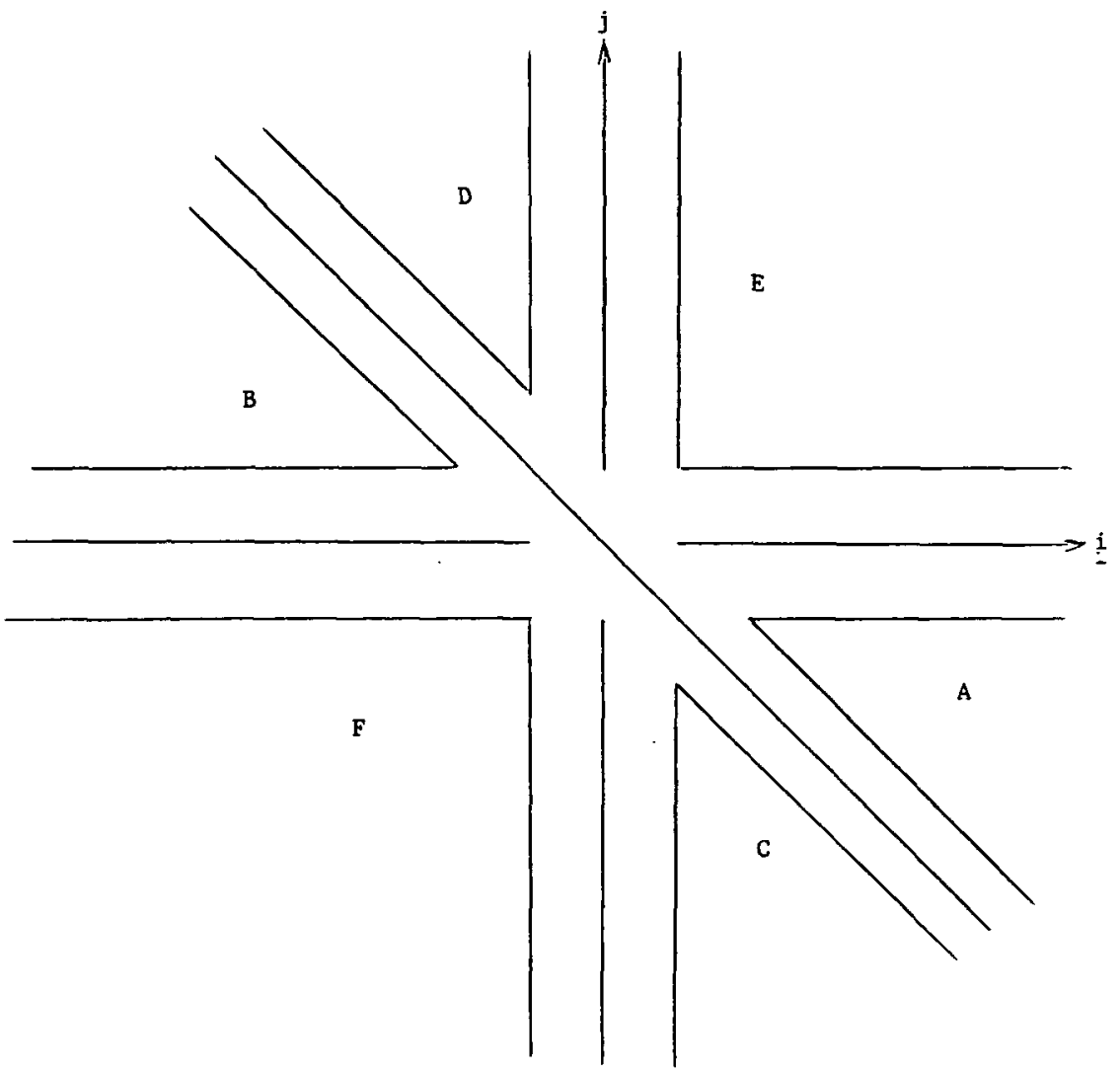

FIGURE 3 Regions for $G_{i j}$.

$$
\left|G_{j}^{i}(t)\right| \leqq\left|\frac{1}{2 j+1} G_{0}^{i}(t)\right|, \quad 0 \leqq t \leqq \frac{1}{2}
$$

and hence from (4.4),

$$
\left|G_{j,-j}\right| \leqq \frac{1}{2 j+1} \cdot \frac{1}{2 j-1} G_{00}, \quad j \neq 0 .
$$

Thus

and so

$$
\sum_{j \neq 0} \frac{\left|G_{j .}-j\right|}{G_{00}} \leqq 2 \sum_{j=1}^{\infty} \frac{1}{2 j+1} \frac{1}{2 j-1}=1
$$

$$
\sum_{-\infty}^{\infty} G_{j,-j}=G_{00}-\sum_{j \neq 0} G_{j,-j} \geqq 0
$$


Now from (4.5) and (4.4) we have

$$
\begin{array}{llll}
G_{2 k-1-l, l}+G_{2 k-l, l} \geqq 0, & k>0, & l<0 & \text { (region A), } \\
G_{2 k+1-l, l}+G_{2 k-l, l} \geqq 0, & k<0, & l>0 & \text { (region B), } \\
G_{k, 2 l+1-k}+G_{k, 2 l-k} \geqq 0, & k>0, & l<0 & \text { (region C), } \\
G_{k, 2 l-1-k}+G_{k, 2 l-k} \geqq 0, & k<0, & l>0 & \text { (region D). }
\end{array}
$$

Following the derivation of $(4.10)$ we have

$$
\begin{array}{cl}
G_{-2 k-1,0}+G_{2 k+1,0}+G_{-2 k-2,0}+G_{2 k+2,0} \geqq 0, & k \geqq 0, \\
G_{0,-2 l-1}+G_{0,2 l+1}+G_{0,-2 l-2}+G_{0,2 l+2} \geqq 0, \quad l \geqq 0, & \\
G_{2 k-1,2 l-1}+G_{2 k, 2 l-1}+G_{2 k-1,2 l}+G_{2 k, 2 l} \geqq 0, \quad k, l>0 & \text { (region E), } \\
G_{2 k+1,2 l+1}+G_{2 k, 2 l+1}+G_{2 k+1,2 l}+G_{2 k, 2 l} \geqq 0, \quad k, l<0 & \text { (region F). }
\end{array}
$$

From (4.20), (4.22)-(4.29) we have $T \geqq 0$.

Case 6. $r$ even, $s$ and $t$ odd. We shall deduce some inequalities for $G_{i j}$. The appropriate regions of $(i, j)$ are illustrated in Figure 4.

We first show that

$$
G_{-1,0}+G_{-2,0}+G_{-2,1}+\sum_{|l|>1} G_{-1, l} \geqq 0
$$

For from (4.3), (4.4) we have for $u, v \in\left[0, \frac{1}{2}\right]$,

$$
\begin{aligned}
& \frac{\left|G_{-2,0}\right|}{G_{-1,0}}+\frac{\left|G_{-2,1}\right|}{G_{-1,0}}+\sum_{|l|>1} \frac{\left|G_{-1,1}\right|}{G_{-1,0}} \leqq \frac{1-u-v}{2-u-v}+\frac{v}{1+v}+\sum_{|l|>1} \frac{v}{|l+v|} \frac{1-u-v}{|l-1+u+v|} \\
& \leqq \frac{1-v}{2-v}+\frac{v}{1+v}+\sum_{2}^{\infty} \frac{v}{l-v} \frac{1-v}{l+1-v}+\sum_{2}^{\infty} \frac{v}{l+v} \frac{1-v}{l-1+v} \\
&=5-\frac{9}{(1+v)(2-v)} \leqq 1 .
\end{aligned}
$$

Similarly we see that

$$
G_{00}+G_{-1,1}+G_{1,0} \geqq 0
$$




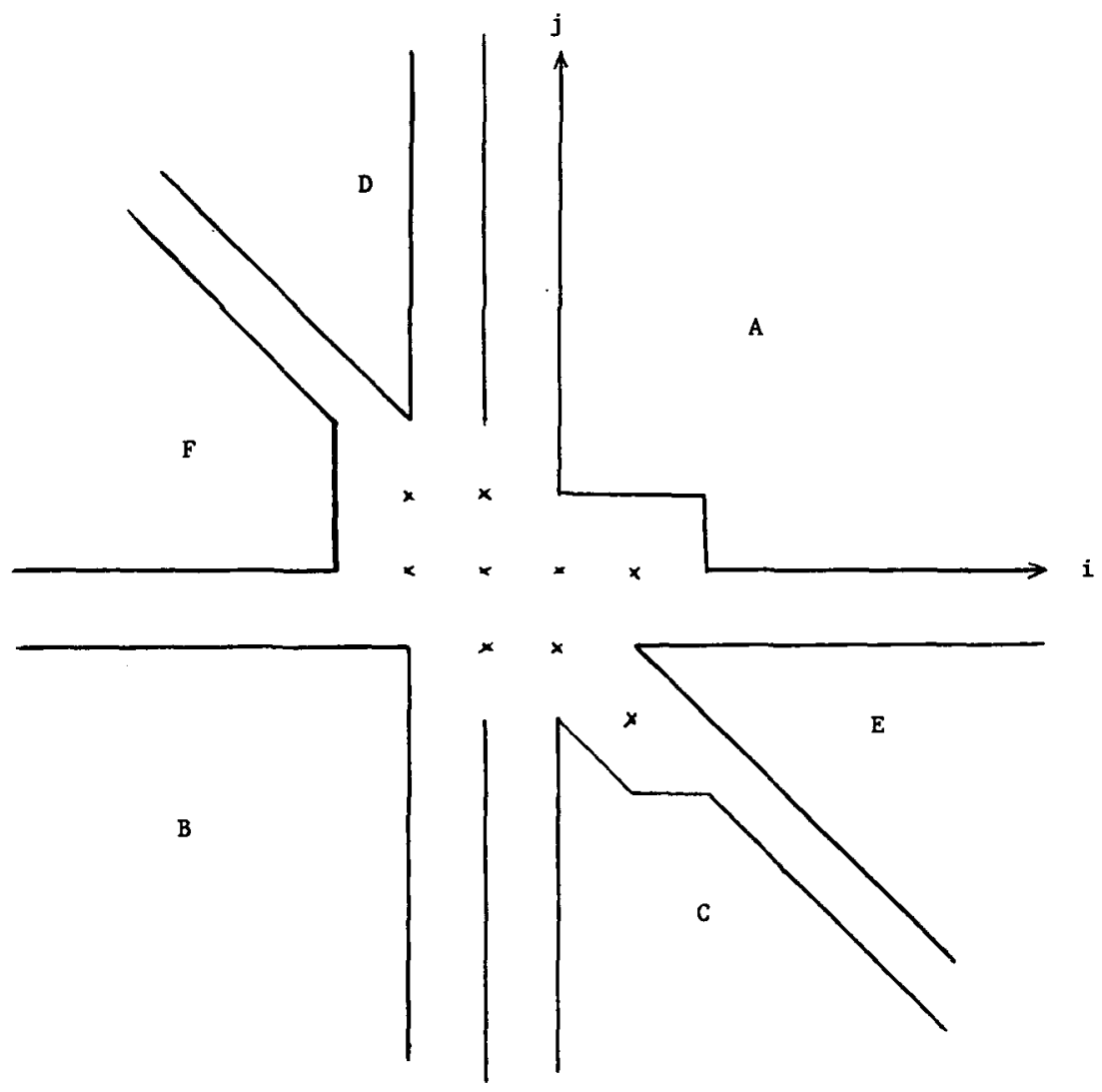

FIGURE 4 Regions for $G_{i j}$.

$$
G_{0,-1}+G_{-1,-1}+G_{1,-2} \geqq 0 \text {. }
$$

Moreover from (4.5) and (4.4) we have

$$
\begin{gathered}
G_{2 k, l}+G_{2 k+l, l} \geqq 0, \quad k, l \geqq 0 \quad(\text { region } \mathrm{A}), \\
\left.G_{2 k+1, l}+G_{2 k, l} \geqq 0, \quad k, l<0 \quad \text { (region } \mathrm{B}\right), \\
\left.G_{2 k, l}+G_{2 k+1, l-1} \geqq 0, \quad k \geqq 0, \quad 2 k+l<0 \quad \text { (region } \mathrm{C}\right), \\
G_{2 k, l}+G_{2 k-1, l+1} \geqq 0, \quad k<0, \quad 2 k+l \geqq 0 \quad \text { (region D), }
\end{gathered}
$$

while following the derivation of (4.10) gives 


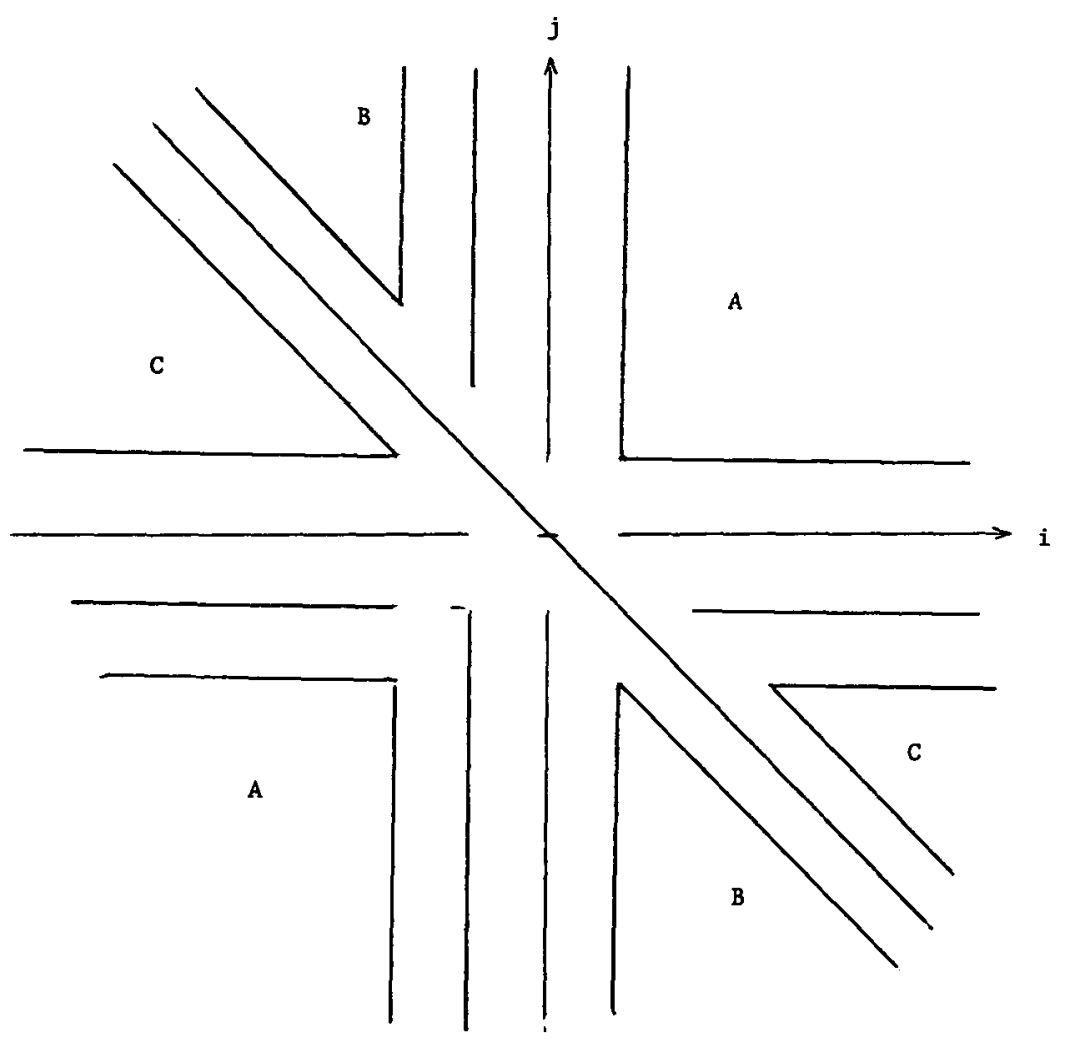

FIGURE 5 Regions for $G_{i j}$.

$$
\begin{array}{llll}
G_{2 k-1,2 l+1}+G_{2 k, 2 l+1}+G_{2 k, 2 l}+G_{2 k+1,2 l} \geqq 0, & l<0, & k+l \geqq 0 \quad \text { (regionE), } \\
G_{2 k+1,2 l}+G_{2 k, 2 l}+G_{2 k, 2 l+1}+G_{2 k-1,2 l+1} \geqq 0, & l \geqq 0, & k+l<0 & \text { (region F). }
\end{array}
$$

From (4.30)-(4.38) we have $T \geqq 0$.

Case 7. $s$ even, $r$ and $t$ odd. This follows from Case 6 by symmetry.

Case 8. $r, s$ and $t$ odd. We shall deduce some inequalities for $G_{i j}$. The appropriate regions of $(i, j)$ are illustrated in Figure 5.

As in (4.30) we have

$$
\sum_{l \neq 1} G_{-1, l} \geqq 0
$$


and similarly

$$
\sum_{|k| \neq 1} G_{k,-1} \geqq 0
$$

From (4.4) and (4.3) we have

$$
\begin{array}{ccc}
G_{k l}+G_{-k-1,-l-1} \geqq 0, & k, l>0 & (\text { region A), } \\
G_{k,-l}+G_{-k-1, l+1} \geqq 0, & 0<k<l & \text { (region } \mathrm{B}), \\
G_{-k, l}+G_{k+1,-l-1} \geqq 0, & 0<l<k & \text { (region } \mathrm{C}) .
\end{array}
$$

Noting also that $G_{k l} \geqq 0$ if $k$ or $l$ is zero and that (4.20) holds as in Case 5, we have $T \geqq 0$.

\section{REFERENCES}

1. C. DE Boor and K. Höllig, B-splines from parallelepipeds, J. Analyse Math. 42 (1982), 99-115.

2. C. DE Boor, K. Höllig and S. RIEMEnSCHNEIDER, Biveriate cardinal interpolation by splines on a three-direction mesh, Illinois J. Math. 29 (1985), 533-566.

3. C. K. Chui, K. Jeeter and J. D. WARd, Cardinal interpolation by multivariate splines, Math. Comp. 48 (1987), 711-724.

4. W. Dahmen and C. A. Micchelli, On the local linear independence of translates of a box spline, Studia Math. 82 (1985), 243-263.

5. A. Ron, Exponential box splines, Constr. Approx., to appear.

Department of Mathematics and Computer Science

UNIVERSITY OF DUNDEE

DUNDEE, DD1 4HN

SCOTLAND 